\title{
A Robust and Dynamic Fire Detection Algorithm using Convolutional Neural Network
}

\author{
K.Sivasankari, Shubham Singh, Kanhaiya Kumar, Aman Dubey
}

\begin{abstract}
The major part of the underlying idea is going to detect the fire from upcoming smoke and the shade color of the smoke using convolutional neural network. The fire detection followed by the smoke detection is going to depend on the shade and the direction vector analysis in this paper. Image processing from the available set of data is very vague ideation so in order to strengthen the idea we are incorporating two main features that is the smoke shade and direction vector. For this major process we will involve data preprocessing through bi-variate hypothesis to select two variables as the color of smoke and the direction of the smoke and hence do the further analysis on other features that how are they going to help in the upcoming detection neurons for the robust algorithm of fire detection

Keywords: Smoke Feature Recognition, Yolo, CNN
\end{abstract}

\section{INTRODUCTION}

It is well known that how much destruction is caused by the fire that sometimes take many hours to extinguish but when it comes to the wildfires this state becomes more hazardous as the wildfires are known to last longer than many days sometimes weeks and months. The wildfires destroy the majority part of the forest cover and the grasslands and that disturbs the whole ecosystem for a long time and the resultant pollution and ash deteriorates the AQI and it takes many years to rejuvenate.

Now let's come to the cause of fire and when we find the causes of majority of wildfires mainly fifty percent of the wildfire reasons are not known to us till date and the other fifty percent is said to have the impact either by the manmade fires or the lightning strikes. So in this case it is impossible to detect the fire unless it is caused so as to figure this down we came to a conclusive idea that if there is a smoke there would be fire and only few features require to be detected and due to that we will be able to detect the fire way before it is caused so that's very important and that would solve the majority of the problems we face while extinguishing, if the precautionary measures taken way before the fire occurs the chances of extreme wild fires reduces by a great percentage and will able to contribute in reducing the carbon footprint also.

Manuscript received on 30 May 2021 | Revised Manuscript received on 07 June 2021 | Manuscript Accepted on 15 June 2021 | Manuscript published on 30 June 2021.

* Correspondence Author

Mrs. K.Sivasankari, Faculty of Computer Science, SRMIST, Bharathi Salai, Ramapuram, Chennai, Tamil Nadu 600089, India

Shubham Singh, Computer Science, SRMIST, Bharathi Salai, Ramapuram, Chennai, Tamil Nadu 600089, India

Kanhaiya Kumar, Computer Science, SRMIST, Bharathi Salai, Ramapuram, Chennai, Tamil Nadu 600089, India

Aman Dubey*, Computer Science, SRMIST, Bharathi Salai, Ramapuram, Chennai, Tamil Nadu 600089, India

(C) The Authors. Published by Lattice Science Publication (LSP). This is an open access article under the CC-BY-NC-ND license (http://creativecommons.org/licenses/by-nc-nd/4.0/)
In the existing system, majority of the fire alarms are either based upon certain chemical reactions or are basically based on the cheap photometry that does not capture or classify images as required hence end up failing and other CCTV devices are not that advance that they can alert for the fire in the bushes and dense forests and they have a certain limitations like they either are using SVM or Bag of Features(BOF) or sometimes CNN or the artificial neural network so as to improve them we would introduce the digital image processing and use classification methods like SVM and feature extraction by the Convolution neural networks because in the recent studies it has shown that if we use a combination of the SVM and the CNN it increases the efficiency and hence the resultant feature extracted is quite efficient.

The data which we usually get and face the major challenge is while distinguishing it. As it is well known that the smoke has no fixed shape or size sometimes we can confuse the smoke through a cloud or fog so in that case we cannot just take the size or the shape data and classify them as the fire causing smoke as this classification would be very inappropriate and will not give a proper algorithm so in order to counter this problem we as discussed earlier will take only two variables that will be the direction vector of the smoke and the shade of the smoke.

The direction vector of the smokes helps us to find that the smoke is either from a fire or is it the fog or the smoke is just a cloud and this classification technique will do the majority of the classification as whenever the smoke is rising from the land and that may be the cause of the fire whenever it is in the upward direction, one major problem that this direction vector solves is that the direction of the fire that where is the fire moving in which direction the fire is moving and that helps the fire departments to deploy the fire trucks or fine men in the non-moving end of the fire so as to save them from the danger and if its possible to grab the data in real time that will suggest the plan changes and improved execution efficacy of the fire department.

The other more advantageous classification would be the shade or the color of the smoke from many studies it is clear that before a fire reaches its burning point smoke has a similar type of the smoke color and that mainly comes out to be the darker colors either the black color or the brownish shades have been proven to give rise to a smoke while the initial stages of the smoke or fire is the lighter smoke colors like the bluish white shades and these are dense as they tend to burn the green leaves first and that provides hydration of the smoke and hence leads to a dense white shades of the smoke. 


\section{PROBLEM DESCRIPTION}

Sometimes when you are training a detector using raw images of object leads to overfitting of model hence reducing overall efficiency and performance of model. Speed of detection and broadcasting medium of SOS can determine the difference between life and death. Placement of sensors used to monitor such an anomaly and resistance to weathering condition and functionality and consistency of device over a period of time can be a crucial factor for timely delivering the distress signal such that it provides enough time for counter measures. Selection of optimal neural network of combination of ANN is very important part of model selection for object detection. Number of input layers training time epochs and learning rate along with optimizer is essential.

Wildlife fire or fire in workplace or residential poses serious risk to life and property of an individual and such incident with preemptive planning can be avoided. A fire needs a dry fuel oxygen supply and ignition temperature. Whenever there is a fire, it starts with dense smoke and fumes. These fumes can be used as indicators. When these datasets are provided to the system there is mainly one issue that we either need to do the preprocessing using multiple variables or we will use only two or three variables so here in the preprocessing task of the data clearing session we came down to the Bi-variate as it is simple to execute and features so to keep it simple we went with the bivariate analysis to get a hypothesis on the data set and the analysis will be the categorical analysis instead of the numerical analysis of bi-variate.

The feature extraction by the CNN should be aided by the SVM as the images pose the problems while feature extraction like the Hughes phenomenon and hence increasing number of bands pose the problem while feature extraction while the SVM is a great method for generalization and is also well reputed at training the data sets any spectral analysis such as the hyperspectral classification can easily be formulated by the formula Fs = $\bigoplus\left\{\sum \mathrm{fs}(\mathrm{I})\right\}$ where the Fs is the fusion feature and the $\oplus$ symbolizes the concatenation vector.

The ResNet allows to form an ANN (artificial neural network) using the cerebral cortex that has more than 150 layers and has an error rate of less than 4 percent. ResNet has a powerful representation and its massive deep neural networks allows for higher precision the major advantage of ResNet was to add the identity shortcuts and the stacking features saves time and also does some reductions in the computations which we require while the feature extraction part and hence the ResNet was involved during the computation of classification and bivariate feature extraction.

The basic MTCNN is based on VG6 and the premiere part was to train the proposal sub-network. The final model is used to initiate the latter part, the detection sub-network was trained with the proposal generates from the proposed sub-network. It is quite identical to adopted SSD, in the proposed subnetwork, we concatenate the chain of domain branches with the similar structure as the detection branches by concatenating two convolutional layers with $4 * 4$ kernel and $6 * 6$ kernels respectively to each scale feature layer also if we take a look at our task we mainly need two

(convo3_3, convo5_4, conv06_1) and 4*4 kernels to the ending feature layer pooling to predict domain category.

\section{LITERATURE SURVEY}

\section{A. Design and Implementation of a CSI-Based Ubiquitous Smoking Detection System}

Ignorance of how to eradicate household smoking in developed countries, existing theory or field-based field techniques cannot provide a specific organization for exposure. With this paper, we undertake the basic goal of building a separate smoking confirmation structure, Smokey, using smoking models based on a Wi-Fi signal to detect smoking activity or within blindness and isolation situations. We read smoker's shows and do the usual highlights to see the development plan during smoking, staying away from the designated ward to prepare for greater accuracy. We plan to get closer to seeing the verification process based on verification to erase basic data from various scattered carriers or influenced by action changes. In addition to the need for target consistency, we move smoking rhythm times to understand smoking tests. We

similarly tracking the quality of various radio cables to assist Smokey's power. Considering the comfort of preparing for new reception lines, Smokey is finally turning to exposing the ubiquitous smoking cessation. We model Smokey with a basic Wi-Fi feature and test its display in real-time mode. Test results show that Smokey is clear and surprising in a variety of contexts. Developmental strategies recognize the repetitive effects of development on remote signals. they are always designed to work wonderfully within the context of fast or clear signals near handsets. It is not clear how unlimited exercise such as smoking is made difficult to access from the handset has an impact on the WiFi signature and whether it is possible to detect smoking with its Wi-Fi signals. In this section, we guide the initial investigation of the discovery of detection of smoking tests using WIFI signals.

The commercial WIFI split provides two channel signals: Received Signal Acquisition Identifier (RSSI) and Channel State Information (CSI). RSSI can be a standard marker that keeps an eye on signal strength. It is OK when human development prevents or opens the signal transmission path, the available signal strength may vary. Fast Smoke Detection Video Surveillance using CUDA

Exposure to smoke can also be a key part of a disaster and a guarantee of safety. Apart from the wide range of smoke and sensory sensitivity techniques proposed, no one has ever chosen to maintain a very high standard while improving manufacturing. With this paper, a smoke detection framework for sensing cameras based on the emergence of smoky environments in addition to concealed data is introduced. philosophy abuses the use of a fixed camera by using the process of restoring the foundation to detect changes within the arena. a shade of smoke is used to determine if it is possible that the pixels inside the scene have a smoky region. due to the changing intensity of the smoke, not all of the certified smoke pixels in the region appear within the hemline of the hemorrhage.

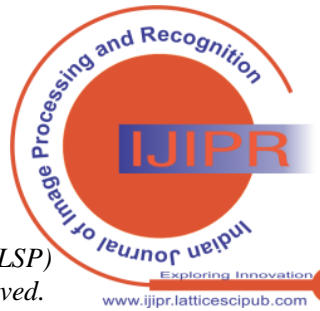


These different pixels are associated with morphological activities and techniques related to the naming component. The presence of a smoke site is acknowledged by violating the negligence of its determination. the final step of the test words is to find the size of the edge pixels within the space. The material interactions within the current and previous edges are designed to block liquid smoke fields from solid articles. A few pieces of testing are maintained using multiple procedures using CUDA AposTherapy which enables quick editing of both unbiased and high-resolution accounts. Calculations have been made for the movement of various videos and show real-time extensions with a limited edge size.

B. Ideal Placement and Intelligent Smoke Detection Algorithm for Wildfire-Monitoring Cameras

Smoke from a rapidly spreading fire is always visible before an explosion. As a result, the initial disclosure of wildfire is important in eliminating the burden of unreasonable assets and critical issues arising from the scorching fire that spreads rapidly. Camera networks are collected and released to detect the smoke circuit of a wildfire. informing the camera's preconceived notions and regional accuracy with a limited financial system, smarter video smarter that separates evidence testing and an excellent system of camera game impact effects during a critical need. With this paper, we suggest that a competent field video format be ready to apply to neighboring cameras. It contains two modules. within the most important module, focused video graphs are held by two nearby models and a light transmitter. within the next module, the main points made are controlled into a simple light bulb at the neural junction, which is a double separator to detect the presence of smoke. In addition to highlighting the rapidly spreading problem of the fire camera as a problem of arranging an equal number of total fire risk of a given area. Parallel testing of real accounts is helped to support the same accuracy due to the computational power of the computer and the memory of the proposed smoke exposure framework. We further validate our proposed camera position by mimicking pre-test camera relationships in a local trial in South California.

C. Adversarial Adaptation from Synthesis to Reality in Fast Detector for Smoke Detection

The video smoke circuit in addition can be a promising fire-retardant strategy. In any case, it has now become a negative aspect of the use of video conferencing sites in real-time confession frameworks, considering how important it is in examining the smoker's image of planning and invisibility of profitable disclosure tests. This paper suggests how fast detectors are stored in the front row, a single box finder, and a large neural convolutional relationship, for smoke detection using smoke imaging tests. Visual data will be able to provide rich models with realworld comments.

Regardless, the discovery of the smoke detection within the identifiers will keep the visual open between verified and deceptive smoke detection, which can cause a significant decrease in the exhibition. training a powerful indicator with smoke tests performed, joining the regional assortment into faster areas. The development of unorganized branches is considered in the context of the design of regional branches into fast-changing areas for change. We are planning a well-structured process of modeling the modified environments, looking at the constant space display of smoke exposure. Spatial segregation, local chaos, and disclosures are integrated within a recurring planning system. The demonstration of the proposed route strikes an important milestone in our evaluation.

\section{SYSTEM ARCHITECTURE}

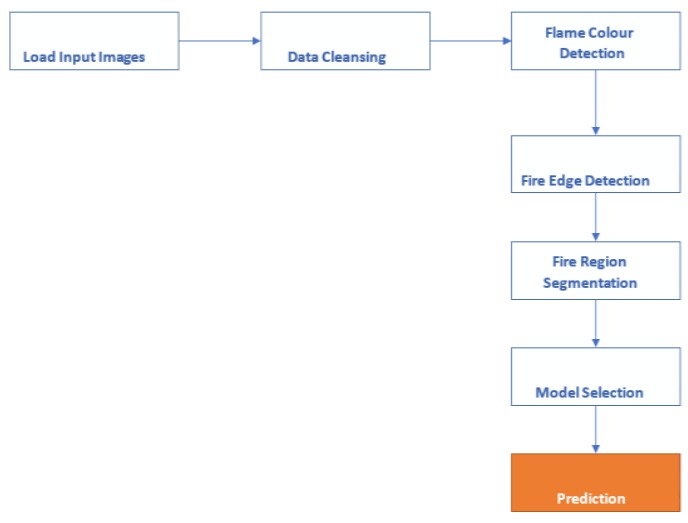

\section{ISSUES IN EXISTING SYSTEM}

- Complexity increases exponentially with data dimension

- Increases computational cost

- Suffers on Statistical Issues

- Highly Unstable

- Produces different clustering results

\section{PROPOSED SYSTEM}

The YOLOv3 model all observable items can be divided into a partial release layer and a yield management layer. The partial release layer can be a combination of Darknet-19 and Res Net-like affiliation, and in addition the facing of the yield layer is similar to the integration of the partial pyramid (FPN). Basic

The layer of layer extraction layer is DBL, as shown in Fig. 3, i.e. the combination of the Convolutional layer, the BN layer (Batch layer in the middle) and the Leaky Re LU layer. It will be able to prevent overcrowding outside the Dropout layer by using the BN layer Compared to the impossible mechanical course on the side of the base station, the Darknet-53 will not have a base of objects on the UAV side. YOLOv3- Relationshipssmall is inserted by the DBL with a layer of model max-pooling, which is used for highlightingto separate the image. in particular, we have added 4 layers of DBL to the minimum releaseincluding a map, which can improve the intensity of small-scale stabilization due to smoke and fire that can be adjusted to normal, can occur in modified sizes. As a result, two layers of YOLO are used to strengthen compliance with the test. Exactly where the data is $416 \times 416$, the critical time crop fragments are $12 \times 11 \times 24$ when the partial map is delivered in the twentieth direction. Also, the second half output is made in layer 27, reducing the yield to $24 \times 24 \times$ 24 . The 24 monitors the tensor size of the relationship.

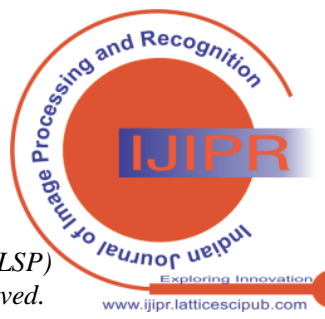




\section{ADVANTAGES OF PROPOSED SYSTEM}

- Reduces Computation Cost for classification tasks

- Reduces Computation Cost for Regression Tasks

- Faster in training the model

- Enhanced generalization by reducing overfitting

- Improved Feature Selection Accuracy

\section{IMPLEMENTATION}

\section{A. Create a CNN Model and Train it}
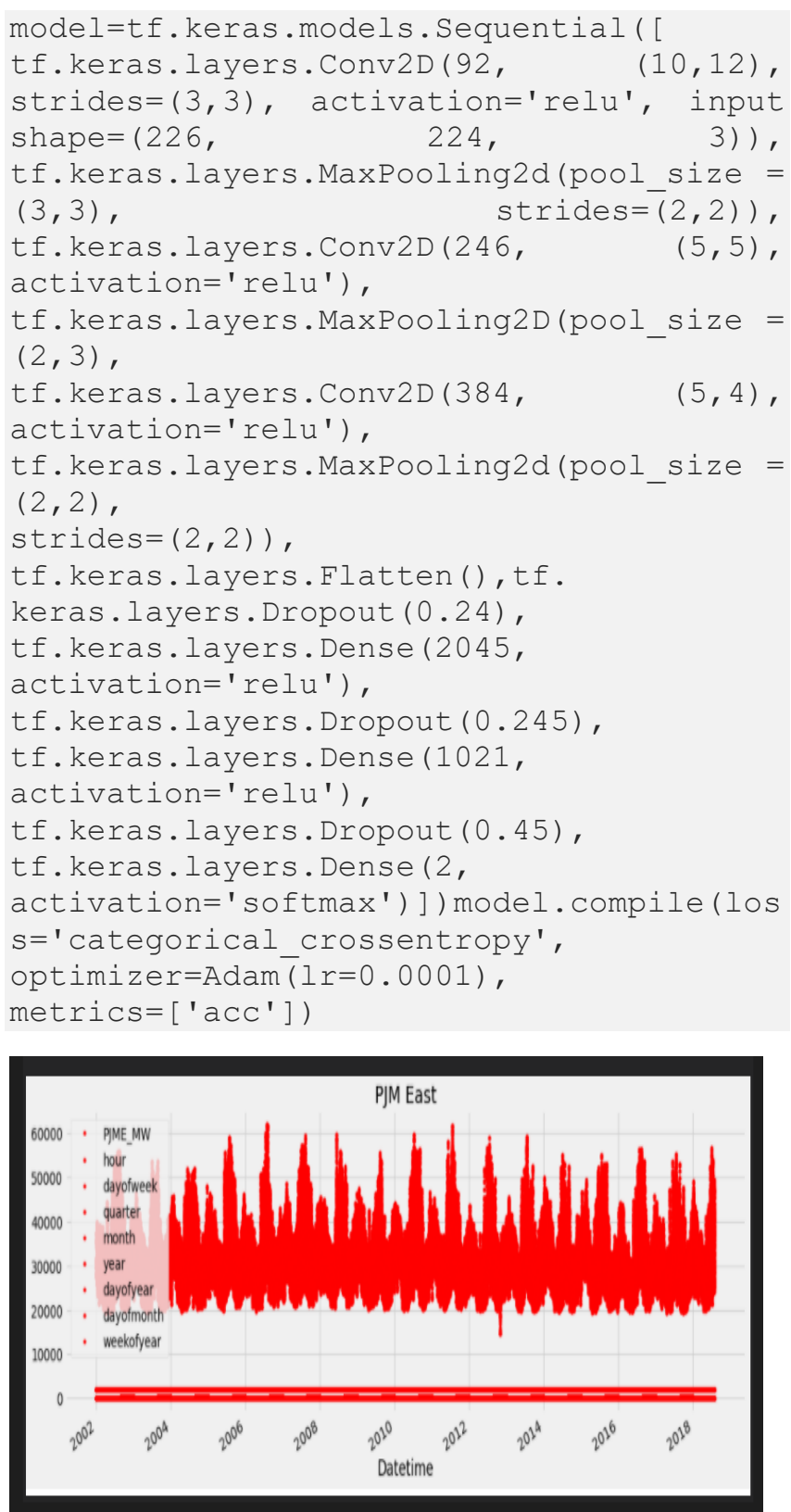

history=model.fit (train_generator, steps per_epoch=6, epochs=10, validation data=vā lidation_generator,validation_stēps=11)

B. In the wake of preparing for 10 ages, we get a preparation exactness of 98.04 and an approval precision of 96.43. The preparation and approval misfortunes are 0.063 and 0.118 individually. Validate the model and test the real time accuracy

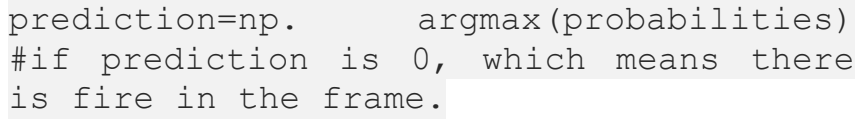

\section{CONCLUSION}

In this program, a basic learning model based on a fire statement based on a small V3 has been introduced. continued with easy development, unlikely regional breakdown, and the provision of additional YOLOv3 additional data, the proposed approach achieves a surprisingly high fire resistance performance with low preparation for a variety of environments and low running time. in the middle of this program, a smoke video process using a ground test is suggested. photos are stored in blocks. the more features of each image block are handled by grids at a smaller co-event level. From an examination of the registration results, we see that these three smoke reflectors have distinct features that do not contain smoke images. The neural backpropagation organization is used for the cessation model. Preliminaries indicate that the proposed framework could detect smoking issues from smokers' accounts that are not in a fast-paced and low-level level of advisors.

\section{REFERENCES}

1. Koubaa and B. Qureshi, "Dronetrack: Cloud-based real-time object trackingunmanned aerial vehicles over the internet," IEEE Access, vol. 6, pp. 13 810- 13 824,2018. [CrossRef]

2. G. R. Ding, Q. H. Wu, L. Y. Zhang, Y. Lin, T. A. Tsiftsis, and Y. D Yao, "An amateur

3. drone surveillance system based on the cognitive internet of things,' IEEECommunications Magazine, vol. 56, no. 1, pp. 29-35, 2018 [CrossRef]

4. Y. LeCun, Y. Bengio, and G. E. Hinton, "Deep learning," Nature, vol. 521, pp. 436-444, May 2015. [CrossRef]

5. Szegedy, A. Toshev, and D. Erhan, "Deep neural networks for object detection," Proc.

6. Int. Conf. Neural Information Processing Systems, vol. 2, pp. 2553 2561, Dec. 2013.

7. R. B. Girshick, "Fast R-CNN," Proc. IEEE Int. Conf. Computer Vision, 2015, pp. 1440-1448. [CrossRef]

8. S. Ren, K. He, R. B. Girshick, and J. Sun, "Faster R-CNN: Towards real-time objectdetection with region proposal networks," Proc. Annual Conf. Neural InformationProcessing Systems, 2015, pp. 91 99.

9. J. Redmon, S. K. Divvala, R. B. Girshick, and A. Farhadi, "You only look once: Unified,real-time object detection," Proc. IEEE Conf Computer Vision and PatternRecognition, 2016, pp. 779-788. [CrossRef]

10. J. Redmon and A. Farhadi, "YOLO9000: Better, faster, stronger," Proc. IEEE Conf.Computer Vision and Pattern Recognition, 2017, pp. 7263-7271. [CrossRef]

11. J. Redmon and A. Farhadi, "YOLOv3: An incremental improvement,"

\section{AUTHORS PROFILE}

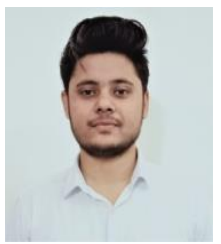

Aman Dubey, Myself Aman Dubey, BTech, Computer Science from SRM University Chennai I am a tech enthusiast who is keen to solve real world problems and that reflects through my projects like Autonomous travelling suitcases to the current research about "Smoke detection through its vector study using ResNet and CNN “. I completed my primary education from Imperial Public School, Varanasi and also posses a strong background in python scripting, Web development and Machine learning and I recently joined TA Digital as a software developer and through my projects and engineering journey I realized that I am quite good at ideation and implementation of various ideas and technologies and wish to solve more such public problems.

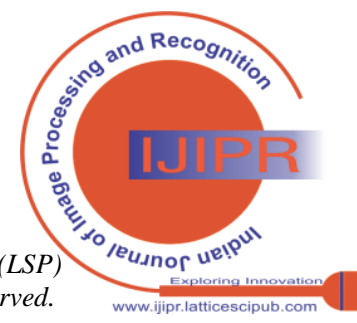




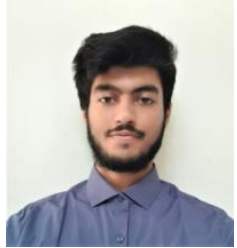

Shubham Singh, My name is Shubham and I am 22 years old. Born and raised in Lucknow, India. Completed my primary education from Delhi Public School. For the past years, I've been studying in SRM University for my Bachelors in Computer Science. I have some background in data analysis, Web development, Game development, Python Scripting with a degree from SRM University. What really got me into the field, though, is the internship I did at Cognizant. Throughout my career, I've noticed that I've always been good with Computers and handling data. Moving forward, I hope to expand my experience across different industries.

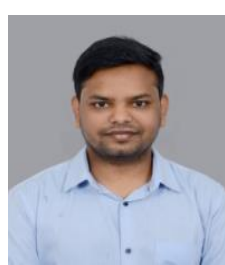

Kanhaiya Kumar, My name is Kanhaiya Kumar and I am 22 years old. Born and raised in Begusarai, India. Completed my primary education from Sri Chaitanya Vidya Niketan. I developed curiosity about the computing technologies that brought me to SRM University Chennai to pursue my bachelors in Computer Science and it was the interest in programming and problem solving that brought me to the research field. I also have a strong background in Web development and Programming. Currently I am interning with Cognizant. Throughout this whole journey, I've noticed that doing what you are good at makes the best out of what you can do.

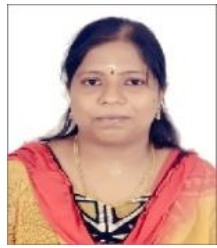

K.Sivasankari, My self Sivasankari. K , working as an Assistant professor in the department of CSE, SRM IST, Ramapuram Campus, Chennai. Completed my HSC in 2002 and my graduation in 2002-04 Anna University and then post graduated from Anna University in 2013. Having 10 years of teaching experience in various engineering colleges and have guided more than 20 batches in my career. I along with teaching subjects from curriculum also trey to inculcate research-based mindset in my students and promote problem solving approach in them that could lead them towards solving some real issues through their knowledge.

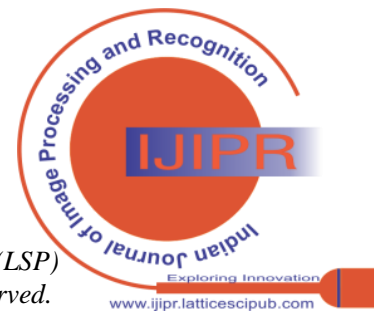

